\title{
The Important Role of Building Provider-Patient Trust in Improving Maternal and Infant Health Outcomes
}

Barb Himes, IBCLC

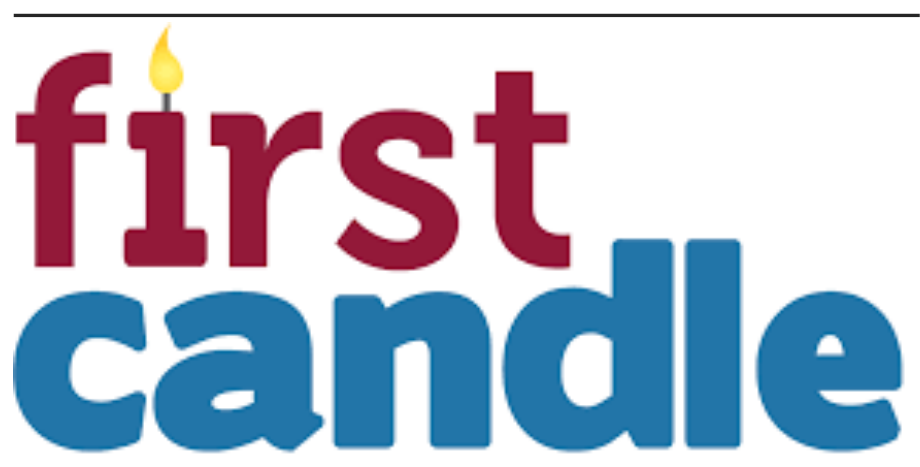

Saving babies. Supporting families.

First Candle's efforts to support families during their most difficult times and provide new answers to help other families avoid the tragedy of the loss of their baby are without parallel.

"We are learning more and more about the fatal effects of racial disparities in U.S. maternal and infant health - the mortality rate of Black mothers in the United States is three to four times higher (42.8 for every 100,000 live births) (1) than their white counterparts - and twice to three times as many Black babies die before their first birthday than do white babies."

We are learning more and more about the fatal effects of racial disparities in U.S. maternal and infant health - the mortality rate of Black mothers in the United States is three to four times higher (42.8 for every 100,000 live births) (1) than their white counter- parts - and twice to three times as many Black babies die before their first birthday than do white babies.

The reasons include disparities in access to quality health care, but they also include racial bias on the part of health care providers, (2) resulting in dismissiveness, false assumptions, and failure to build trust. As we have previously reported, Black women in the U.S. can experience "an inescapable atmosphere of societal and systemic racism [that] can create a kind of toxic physiological stress, resulting in conditions - including hypertension and pre-eclampsia - that leads directly to higher rates of infant and maternal death." (3)

The issue of trust leads to the critical flip side: Black newborns have a greater chance of surviving childbirth if their physicians are Black doctors, according to a 2019 study (4). Its authors note that the disparity is likely the effect of structural racism, a "social construct that has gotten under the skin." (5)

The evidence suggests racial concordance between health care providers and patients can lead to relationships freer from stress, fears, and maternal concerns about being dismissed, resulting in relationships of greater trust between the provider and patient.

But there is a problem: According to the Association of American Medical Colleges, only five percent of American doctors identify as Black. Dr. Valerie Montgomery Rice, dean of the Morehouse School of Medicine, a historically Black medical school, believes that healthier outcomes depend in part on:

- A patient's trust in a doctor

- A positive doctor-patient relationship

- An understanding of the patient's environment

She notes this can occur "through a cultural lens. If you can relate to something about that person's story or have some indication of what their experience has been, then the recommendations you make as a provider are going to make a difference."

In light of this need, Morehouse and CommonSpirit Health have recently announced plans to increase the U.S. Black physician population by launching five Morehouse regional medical school campuses in underserved communities that also have CommonSpirit facilities and by recruiting students from provider-deprived

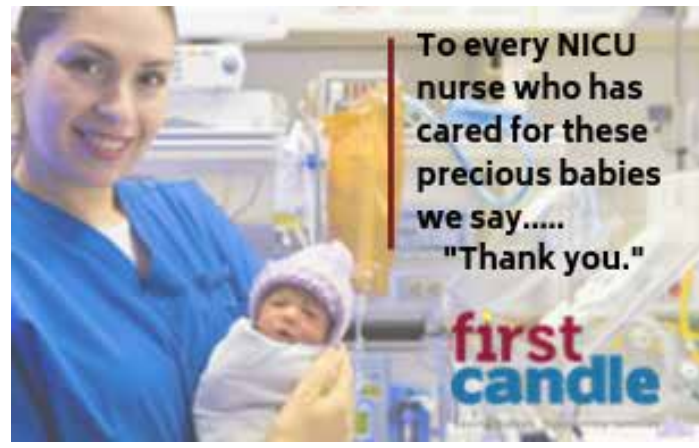

\section{Did you know that premature and low birth weight babies have a $4 \times$ greater risk for SIDS?}

At First Candle we're educating parents, grandparents and caregivers about safer sleep to make sure all babies reach their first birthday. Learn more at firstcandle.org 
communities. The curriculum will include cultural competency in patient care. (6)

This is a heartening and overdue step that needs to be built upon, but it is also important to face the reality that the current majority of health care providers is likely not to be Black. This, however, does not mean building greater provider-patient trust cannot be achieved and that it should not be strongly pursued. There are steps that can and should be taken.

At First Candle, our Straight Talk for Infant Safe Sleep training program actively explores unconscious bias and works with care providers to improve communication with patients, to better understand their real-world obstacles and objections to adopting safe sleep guidelines and breastfeeding, which can significantly reduce the rates of infant mortality. The awareness raised with Straight Talk participants and the learnings from these sessions can positively affect the providers' work to reduce sleep-related infant mortality and influence overall approaches to patient-centered care.

\section{"The aim is for providers to develop and demonstrate an ability to listen, learn, and engage in respectful dialogue with families. Providers must engage themselves as they look for ways to engage their patients."}

The aim is for providers to develop and demonstrate an ability to listen, learn, and engage in respectful dialogue with families. Providers must engage themselves as they look for ways to engage their patients. How do I feel about my patient and her pregnancy journey, before, during, and after? What beliefs and experiences do I bring to this relationship of care?

It is important to reach patients "where they are", where they live and feel.

- Assume good intent

- Be open

- Consider another perspective

- Word choice is important

- $\quad$ Listen, pause, reflect

This includes looking at your own assumptions, which could range from gender (doctors are males, nurses are females) to racial and socioeconomic stereotyping. The intent should be to respect your patient's and her family's wishes and needs, remembering that each family is unique and that bias exists.

Another area of sensitive engagement may include Adverse Child Experiences (ACE). Our childhood experiences have a significant lifelong impact on our health and the quality of our lives. ACE has been linked to risky behavior, psychological issues, serious illness, and even death. Some ACEs are childhood abuse, neglect, and household challenges. Being aware of potential
ACEs can help the provider to consider home visiting programs, parenting training, domestic violence prevention, mental illness and substance abuse treatment, quality childcare availability, and community support for parents.

The provider-patient relationship is an opportunity to practice active listening to understand what patients are truly saying.

- Consciously work to build trust and establish rapport.

- Ask specific questions, which can also be reflective in nature: What do you think about this? How do you feel about this?

- Include verbal affirmations in your conversations (I understand; I see).

- Show interest and concern.

This evaluation extends to the physical environment: Is the waiting room and its staff welcoming and inclusive? In a meeting, is the provider looming over the patient, or are they on equal footing? If it is a telehealth video session, is the provider lit and positioned to be visually approachable and conversational? Is the patient's partner invited to participate actively?

\section{“The practitioner's medical knowledge and experience is the foundation for sustaining maternal and infant health, and his or her interpersonal understanding and true desire to foster a trusting relationship can further that knowledge, to result in improved health outcomes for mothers and babies of all ethnicities, gender identifications, and circumstances."}

The practitioner's medical knowledge and experience is the foundation for sustaining maternal and infant health, and his or her interpersonal understanding and true desire to foster a trusting relationship can further that knowledge, to result in improved health outcomes for mothers and babies of all ethnicities, gender identifications, and circumstances.

\section{References:}

1. CDC Vital Signs, May 2019

2. Racial bias in pain assessment and treatment recommendations, and false beliefs about biological differences between blacks and whites. Proceedings of the National Academy of Sciences, April 4, 2016.

3. Why America's Black Mothers and Babies Are in a Life-orDeath Crisis. New York Times, April 11, 2018

4. Physician-patient racial concordance and disparities in birthing mortality for newborns. Greenburg et al. Proceedings of the National Academy of Sciences, September 1, 2020, vol. 117, no. 35.

5. Black babies are more likely to survive when cared for by 
Black doctors, study finds. USA Today, August 19, 2020.

6. America has an urgent need for black doctors: 'It's hard to be what you can't imagine.' USA Today, December 26, 2020.

\section{About First Candle}

First Candle, based in New Canaan, CT, is a 501c (3) committed to eliminating Sudden Infant Death Syndrome and other sleeprelated infant deaths while providing bereavement support for families who have suffered a loss. Sudden unexpected infant death (SUID), which includes SIDS and accidental suffocation and strangulation in bed (ASSB), remains the leading cause of death for babies one month to one year of age, resulting in 3,600 infant deaths nationwide per year.

Disclosure: The author is the Executive Director and Chief Executive Officer of First Candle, Inc., a Connecticut not for profit 501c3 corporation.

NT

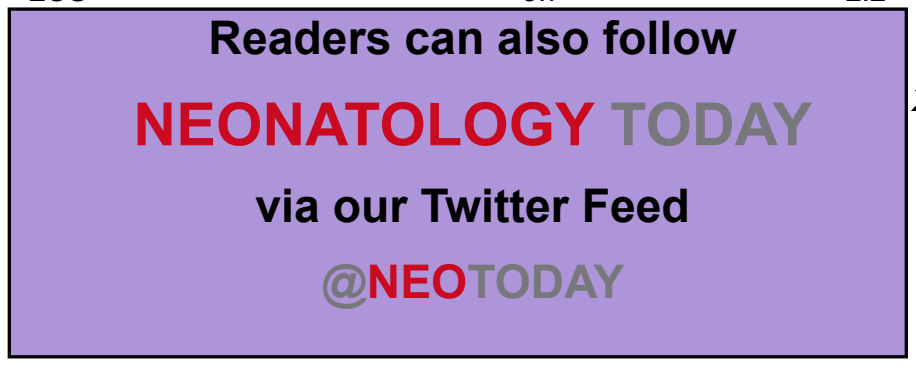

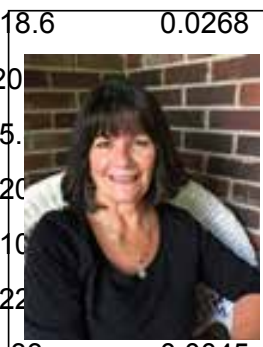

Corresponding Author

Barb Himes, IBCLC

Director of Education and Bereavement Services

First Candle

49 Locust Avenue, Suite 104

New Canaan CT 06840

Telephone: 1-203-966-1300

For Grief Support: 1-800-221-7437

barb@firstcandle.org

www.firstcandle.org

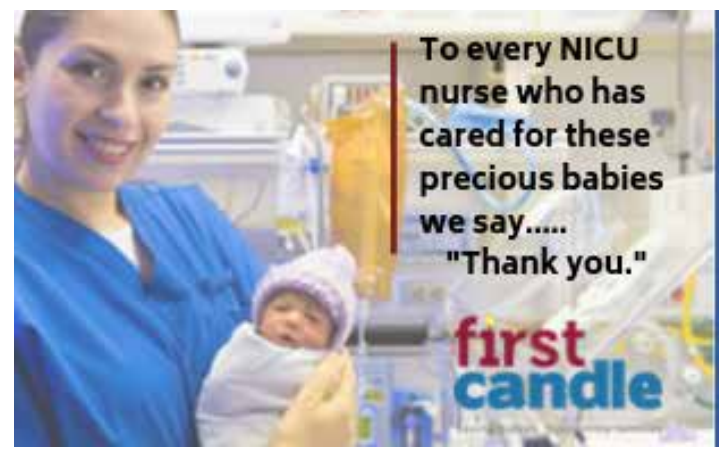

\section{Did you know that premature and low birth weight babies have a $4 x$ greater risk for SIDS?}

At First Candle we're educating parents, grandparents and caregivers about safer sleep to make sure all babies reach their first birthday. Learn more at firstcandle.org
The National Urea Cycle Disorders Foundation

Time is precious, just like your patients.
The NUCDF is a non-profit organization dedicated to the identification, treatment and cure of urea cycle disorders. NUCDF is a nationally-recognized resource of information and education for families and healthcare professionals. 